\title{
Measurements of Intrinsic Ion Bernstein Waves in a Tokamak by Collective Thomson Scattering
}

\author{
S. B. Korsholm, ${ }^{1, *}$ M. Stejner, ${ }^{1}$ H. Bindslev,${ }^{1}$ V. Furtula, ${ }^{1}$ F. Leipold, ${ }^{1}$ F. Meo, ${ }^{1}$ P. K. Michelsen, ${ }^{1}$ D. Moseev, ${ }^{1}$ \\ S. K. Nielsen, ${ }^{1}$ M. Salewski, ${ }^{1}$ M. de Baar, ${ }^{2}$ E. Delabie, ${ }^{2}$ M. Kantor, ${ }^{2,3}$ A. Bürger, ${ }^{4}$ and TEXTOR Team ${ }^{4}$ \\ ${ }^{1}$ Association EURATOM-Risф National Laboratory for Sustainable Energy, Technical University of Denmark, \\ DK-4000 Roskilde, Denmark \\ ${ }^{2}$ FOM-Institute for Plasma Physics Rijnhuizen, Association EURATOM-FOM, PO Box 1207, 3430 BE Nieuwegein, The Netherlands \\ ${ }^{3}$ Ioffe Institute, RAS, St. Petersburg 194021, Russia \\ ${ }^{4}$ Institute for Energy Research, Plasma Physics, Forschungszentrum Jülich GmbH, Association EURATOM-FZJ, \\ D-52425 Jülich, Germany
}

(Received 11 November 2010; published 20 April 2011)

\begin{abstract}
In this Letter we report measurements of collective Thomson scattering (CTS) spectra with clear signatures of ion Bernstein waves and ion cyclotron motion in tokamak plasmas. The measured spectra are in accordance with theoretical predictions and show clear sensitivity to variation in the density ratio of the main ion species in the plasma. Measurements with this novel diagnostic demonstrate that CTS can be used as a fuel ion ratio diagnostic in burning fusion plasma devices.
\end{abstract}

Waves and particle dynamics in the frequency range of ion cyclotron motion (ICM), $\omega_{c i}=q_{i} B / m_{i}$, play important roles for diagnostic of ion composition in magnetized plasmas. Under certain conditions collective Thomson scattering (CTS) spectra are distinctively sensitive to ion Bernstein waves (IBWs) and the ICM. The combined effect of IBWs and ICM gives rise to an ion cyclotron structure (ICS) in CTS spectra which depend on the ion species densities and their ratios. Measurements of the ICS in CTS spectra can thus demonstrate basic plasma and wave physics as well as provide great diagnostic potential for characterizing the composition of magnetized plasmas.

We present in this Letter the first measurements of CTS spectra with signatures of intrinsic IBWs and ICM in a multispecies tokamak plasma. In experiments on multispecies plasmas containing, e.g., hydrogen, deuterium, and ${ }^{3} \mathrm{He}$, we demonstrate that we resolve changes in the ICS in CTS spectra caused by variations in the isotope ratios. In addition, calculations and experimental results agree. This indicates that the relevant physics of CTS and the interaction with IBWs and ICM are well represented by our kinetic, fully electromagnetic model.

While our results are of general interest and have application in plasma physics, there is a particular implication on the field of fusion energy research. The issue of measuring the fuel ion ratio in future burning plasma magnetic confinement devices has recently received increasing attention. The fuel ion ratio in burning plasmas is the ratio of the number densities of the main ion species. On ITER it is not clear if this ratio can be determined in the plasma center (normalized radius $\rho<0.3$ ) with the diagnostic set currently included in the ITER baseline design [1]. As measurements of the fuel ion ratio are of high priority for machine protection and basic control, it is of great interest to develop alternative diagnostic methods capable of measuring the tritium-deuterium ratio. Our results represent a key step for developing a novel CTS-based fuel ion ratio diagnostic which can fulfill the measurement needs for ITER and future devices.

IBWs are electrostatic hot plasma waves generally present in magnetized plasmas [2,3]. IBWs are weakly damped for propagation near perpendicular to the magnetic field $\left(k_{\|} \approx 0\right)$ while the damping of the waves increases strongly as $k_{\|}$increases. The frequencies of propagating IBWs lie between the harmonics of the cyclotron frequency for each ion species in the plasma, $\omega_{c i}$. The frequencies of IBWs with wave vectors relevant to our measurements lie close to the ion cyclotron harmonics. Their frequency separation is therefore roughly the cyclotron frequency of the plasma ions, and each IBW can be related to the presence of ion species with specific $\omega_{c i}$. When resolving $k_{\|} \approx 0$, IBW signatures will appear in CTS spectra as multiple peaks with frequency separation of $\sim \omega_{c i}$ for each plasma species. For low- $k_{\|}$geometries ICM will also cause peaks in CTS spectra. When $k_{\|}$ increases, these peaks will broaden and effectively disappear. The peaks will appear at the ion cyclotron harmonics. While the frequency separations in each class of signatures are $\sim \omega_{c i}$, the IBWs are generally not spectrally coincident with harmonics of the ion cyclotron frequency. In CTS spectra with $k_{\|} \approx 0$ the signatures are generally both due to effects of IBWs and ICM [4]. The resulting ICS is more elaborate than simply the superposition of the two effects. Species with the same charge to mass ratio give rise to IBW and ICM signatures in CTS spectra with the same frequency separation. However, the contributions to CTS spectra due to individual species are also dependent on their masses, since the Doppler shift $\left(\omega^{\delta} \propto \mathbf{v}_{\text {ion }} \cdot \mathbf{k}^{\delta}\right)$ is smaller for the bulk ion population of the heavier ions if the ion species are in thermal equilibrium. Hence, it is in 
theory possible to distinguish the contributions due to, e.g., populations of deuterium and helium. Additionally, as ICS in CTS spectra due to a given species are strongly dependent on its number density, it is in principle possible to infer an isotope or fuel ion ratio from measured CTS spectra.

Measurements of IBWs to determine minority ion concentrations in weakly ionized hydrogen gases have been made using probes in the Thorello and Blaamann devices, respectively [5,6]. Measurements with far-infrared CTS of externally excited IBWs in plasmas were done at Microtor [7,8], ACT-1 [9], and Alcator-C [10] where the wave dispersion of an IBW was obtained in shot-by-shot scans of the scattering angle, i.e., for different wave vectors. Gyrotron-based measurements of IBW signatures in CTS spectra was demonstrated on the Tara Tandem Mirror axicell during ion cyclotron resonance frequency heating [11]. Richards et al. proposed to measure the fuel ion ratio in ITER by measuring the IBW spectrum using a $\mathrm{CO}_{2}$ laser-based CTS diagnostic [12]. Preliminary calculations indicated that the fuel ion ratio could in principle be determined with such a system.

The aim of the proof-of-principle CTS-based fuel ion ratio diagnostic is to demonstrate that CTS spectra contain information on ICM and intrinsic IBWs (i.e., without external drive) under relevant tokamak plasma conditions and that the spectra are sensitive to changes in the isotope content of the plasma.

A CTS system provides temporally resolved and spatially localized information on confined ions projected along a given direction. The CTS spectra are obtained by injecting a powerful beam of electromagnetic waves into the plasma. The incident wave $\left(\mathbf{k}^{i}, \omega^{i}\right)$ scatters off microscopic fluctuations in the plasma. At scales larger than the Debye length, these microscopic fluctuations are primarily due to ion dynamics [13]; hence the incident wave scatters off fluctuations which are the plasma collective response to moving ions. A portion of the resulting scattered radiation $\left(\mathbf{k}^{s}, \omega^{s}\right)$ is picked up by a receiving aperture defining the receiver beam. The spatial localization is determined by the overlap between the probe and receiver beams, and the resolved wave is defined by $\left(\mathbf{k}^{\delta}, \omega^{\delta}\right)=\left(\mathbf{k}^{s}, \omega^{s}\right)-$ $\left(\mathbf{k}^{i}, \omega^{i}\right)$. From the spectrum of the received scattered radiation, one may infer the 1D projection along $\mathbf{k}^{\delta}$ of the full ion velocity distribution function. This is used for CTS measurements of fast-ion dynamics, such as those of the CTS diagnostic at TEXTOR [14-16].

At geometries where $\mathbf{k}^{\delta}$ is close to perpendicular to the main magnetic field $\mathbf{B}, \phi=\angle\left(\mathbf{k}^{\delta}, \mathbf{B}\right) \approx 90^{\circ}$, the CTS spectrum becomes sensitive to IBWs [17]. The relevant plasma fluctuation spectrum under such conditions has a different physical origin from that of the fast-ion induced fluctuations. In contrast to the case of the fast-ion induced fluctuations, it depends on the presence of a magnetic field through the ICM and modifications to the plasma dielectric response via IBWs [4]. In Fig. 1 we show the calculated spectral power densities for $\phi=93^{\circ}$ and for $\phi=120^{\circ}$,

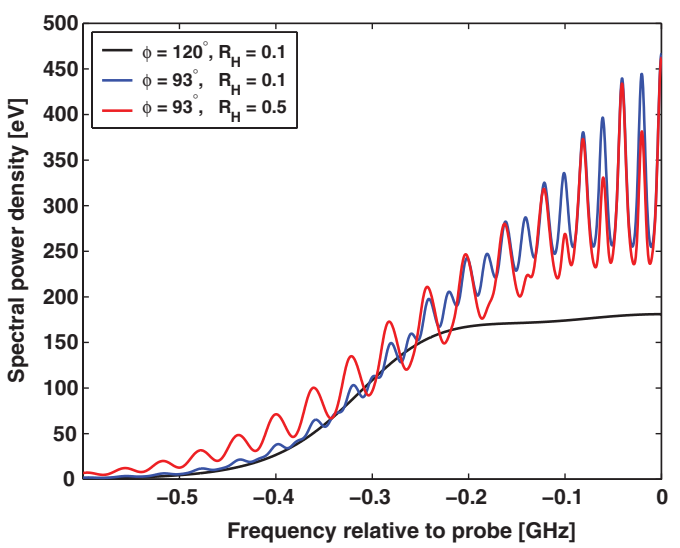

FIG. 1 (color). Calculated CTS spectra for a geometry with $\mathbf{k}^{\delta}$ close to perpendicular to $\mathbf{B}, \phi=93^{\circ}$ in a typical TEXTOR plasma for $R_{\mathrm{H}}=0.1$ (blue) and $R_{\mathrm{H}}=0.5$ (red). For comparison a calculated CTS spectrum for $\phi=120^{\circ}$ (black) and $R_{\mathrm{H}}=0.1$.

i.e., close to and far from perpendicular, respectively. The peaks of the ICS are only present in the spectra for $\phi=93^{\circ}$. The spectra are calculated for typical TEXTOR plasma parameters for two different ratios of the $\mathrm{H}$ and $\mathrm{D}$ number densities. Here we define the fuel ion (or rather isotope) ratio as: $R_{\mathrm{H}}=n_{\mathrm{H}} /\left(n_{\mathrm{H}}+n_{\mathrm{D}}\right)$. From the curves for $\phi=93^{\circ}$, it is apparent that the ICS in the CTS spectrum depend significantly on the isotope ratio; thus the measured CTS spectra should show sensitivity to changes in the isotope content. However, CTS spectra are generally dependent on several other plasma parameters, and inference of the isotope ratio from the data is challenging. In particular, the spectra depend strongly on the angle $\phi$ and the ion temperature $T_{i}$. Therefore, in order to limit the uncertainty in the determination of the isotope ratio, accurate knowledge of the scattering geometry and plasma parameters is important.

In the analysis of the measured spectra and for the calculated spectra (as in Fig. 1), we use a fully electromagnetic model accounting for the interaction between the incident electromagnetic wave and the microscopic fluctuations in the density, magnetic field, electric field, and electron current density $[17,18]$.

The measurements were performed on the TEXTOR tokamak. The experimental setup for the CTS diagnostic at TEXTOR is described in detail in Ref. [19]. The source for the probing beam is a $110 \mathrm{GHz}$ gyrotron with a power of $150 \mathrm{~kW}$ and a maximum integrated pulse length of $200 \mathrm{~ms}$. The probe and the receiver beams are transmitted via quasioptical transmission lines coupling to the plasma via steerable mirrors allowing for flexible choice of scattering geometry. The received scattered radiation is transmitted to a sensitive heterodyne receiver protected against gyrotron stray light by a set of narrow notch filters. For the present measurements, a portion of the central part of the spectrum is fed into a separate second heterodyne downconversion step. By this procedure the desired part of the spectrum can be acquired with an acquisition card at 
$5 \times 10^{9}$ sample $\mathrm{s}^{-1}$. Further details on the diagnostic setup, the measurement procedure, and the signal analysis are described in Ref. [20].

To investigate the effect of scattering geometry on the ICS in CTS spectra, the resolved angle $\phi$ was changed during one discharge. This was achieved by rotating the receiving and probing antennae mirrors in tandem while maintaining the overlap between the beams. In Fig. 2, we present the results of the experiment by seven representative spectra with resolved angles around $90^{\circ}$. For directions near perpendicular to $\mathbf{B}$ the ICS in CTS spectra become clear while they gradually disappear for propagation directions away from perpendicular. The experimental results summarized in Fig. 2 establish that the diagnostic system is sensitive to ICS and demonstrate the sensitivity to the resolved angle.

In a series of experiments the effect of the isotope ratio on the ICS was investigated in plasmas consisting of hydrogen, deuterium, and/or ${ }^{3} \mathrm{He}$. The results are summarized as three representative curves in Fig. 3. The isotope ratio was changed from $R_{\mathrm{H}} \approx 0.1$ (deuterium rich, discharge no. 109126) to $R_{\mathrm{H}} \approx 0.4$ (discharge no. 110416). The estimates of $R_{\mathrm{H}}$ have been obtained from the plasma edge by passive spectroscopy [21]. In discharge no. 109126 the frequency spacing between the peaks is approximately $20 \mathrm{MHz}$ corresponding to the ion cyclotron frequency of deuterium at the location of the scattering volume $\left(B_{t} \approx 2.55 \mathrm{~T}\right)$. By close inspection, one may see that every

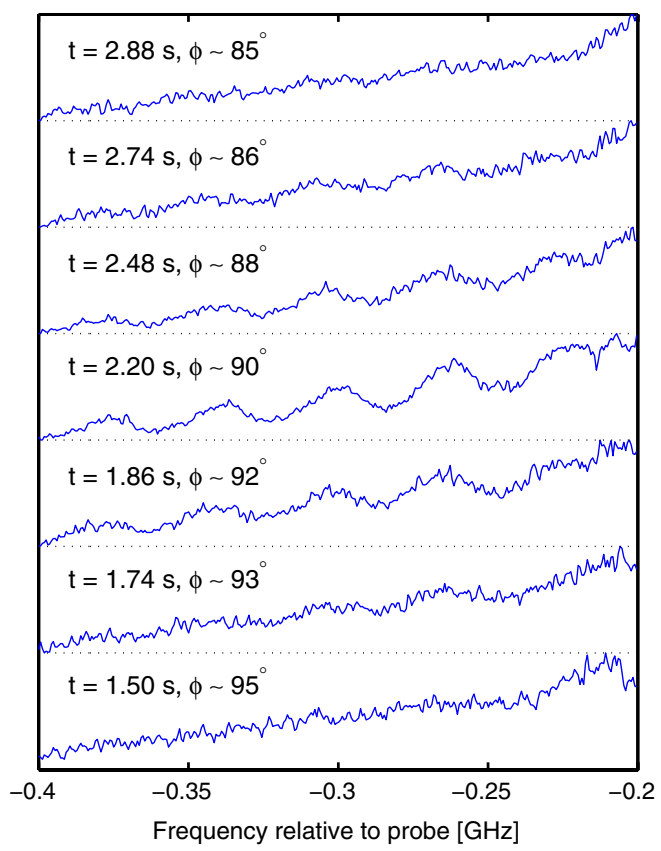

FIG. 2 (color online). Measured CTS spectra for seven representative angles around $\phi \approx 90^{\circ}$ made during a sweep of the probing and receiving mirrors during TEXTOR discharge no. 111796 . The spacing between the peaks is $40 \mathrm{MHz}$, corresponding to $\omega_{c \mathrm{H}}$ at the location of the scattering volume. The respective time and approximate $\phi$ are indicated in each graph. The $\phi$ values are obtained by ray tracing. second peak has a larger relative amplitude than the neighboring peaks (adjusting for the general slope of the spectrum). This is in accordance with the expectation that the ICS modulation related to hydrogen would add to every second peak since $\omega_{c \mathrm{H}}=2 \omega_{c \mathrm{D}}$. As we decrease the relative deuterium content in the plasma, the peaks exclusively due to deuterium effectively disappear. In the spectrum of discharge no. 110416 the remaining dominant peaks have a frequency spacing of approximately $40 \mathrm{MHz}$, corresponding to the ion cyclotron frequency of hydrogen. From the modeled spectrum for $R_{\mathrm{H}}=0.5$ in Fig. 1, one may see that in the frequency range outside the notch filter $(f<-0.15 \mathrm{GHz})$ one would not expect to see clear peaks originating from deuterium when approaching an equal mixture of $\mathrm{H}$ and D. The results in Fig. 3 are consistent with this. However, while not clearly visible, the ICS due to deuterium still contributes to the spectral power density, and the spectrum remains sensitive to the isotope ratio.

In a series of discharges, the plasma was fueled by ${ }^{3} \mathrm{He}$, and shot-by-shot the ratio of ${ }^{3} \mathrm{He}$ was increased. The spacing between the dominant peaks in the spectrum of discharge no. 110421 (Fig. 3) is approximately $26 \mathrm{MHz}$, corresponding well to the ion cyclotron frequency of ${ }^{3} \mathrm{He}$ at the magnetic field in the scattering volume. This demonstrates a clear sensitivity of the diagnostic to the isotope content of the plasma.

In Fig. 4 we present measured and calculated spectra for TEXTOR discharge discharge no. 109155, which was heated by neutral beam injection (NBI). The plasma parameters $T_{i}, T_{e}$, and $n_{e}$ for the calculation are given by the measured values from charge exchange recombination spectroscopy and incoherent Thomson scattering. We assume an equal mixture of $\mathrm{H}$ and $\mathrm{D}\left(R_{\mathrm{H}}=0.5\right)$ for the calculation, lacking other diagnostics for the isotope mixture in the plasma core. This is reasonable for a deuterium fueled plasma with hydrogen NBI. In the lower part of Fig. 4 we plot the relative differences between the measured and calculated spectra for $R_{\mathrm{H}}=0.25,0.5,0.75$. We

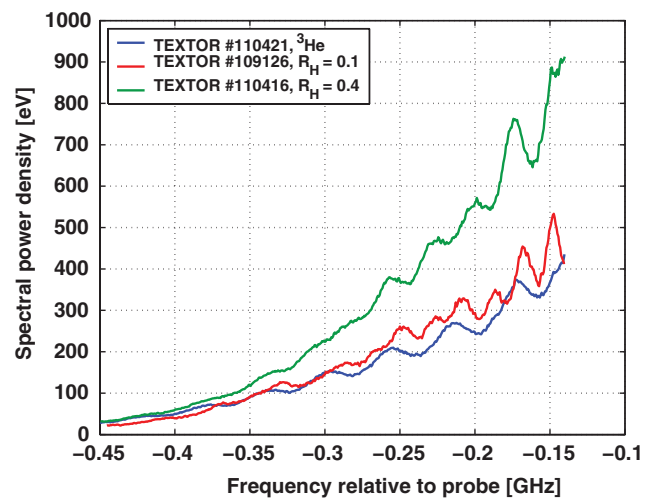

FIG. 3 (color). Measured CTS spectra in TEXTOR for a ${ }^{3} \mathrm{He}$ fueled plasma (green, discharge no. 110421), a deuterium dominated plasma (red, discharge no. 109126) and a plasma with $40 \%$ hydrogen (blue, discharge no. 110416). The spacing between the ICS peaks corresponds to the ion cyclotron frequencies of ${ }^{3} \mathrm{He}$, $\mathrm{D}$, and $\mathrm{H}$, respectively. 

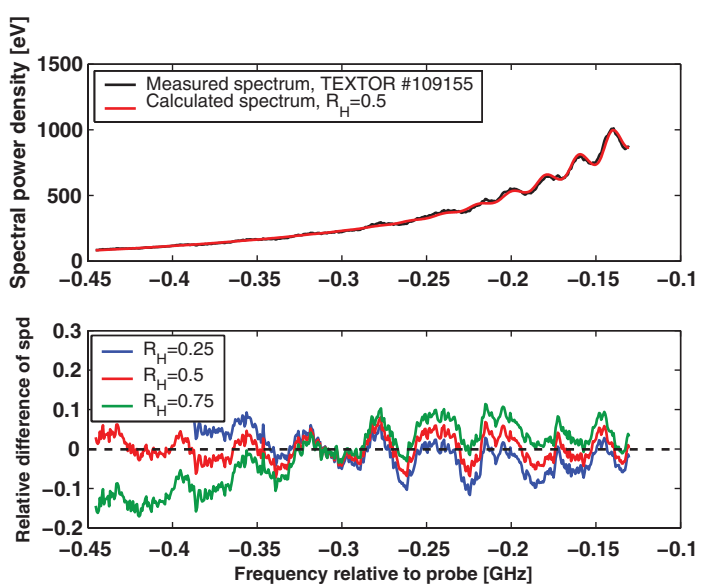

FIG. 4 (color). Measured and calculated CTS spectra for TEXTOR discharge no. 109155 (top). $n_{e}$ and $T_{e}$ used in the calculations are from Thomson scattering while $T_{i}$ is from the charge exchange diagnostic. The calculated spectrum are shown for $R_{\mathrm{H}}=0.5$. Relative differences between measured and calculated spectra for $R_{\mathrm{H}}=0.25,0.5,0.75$ (bottom).

note that the graph for $R_{\mathrm{H}}=0.5$ has the smallest amplitude and oscillates around zero and hence is closest to the experimental value of $R_{\mathrm{H}}$. The measured spectrum is well reproduced by the theoretical spectrum. This gives confidence that the model encompasses the relevant physical mechanisms and quantities to describe the involved physics, which is a prerequisite for the ability to infer the isotope ratio from measured spectra.

The results presented here are the first measurements of CTS spectra with signatures of intrinsic IBWs and ICM in a tokamak plasma. As predicted by theory, the experimental investigations have found ICS modulated CTS spectra to be sensitive to plasma composition and scattering geometry. The frequency separation of the measured ICS peaks in the spectra is dependent on the cyclotron frequency of the main isotopes in the plasma consistent with theory. Hence, despite sensitivity to other parameters, the diagnostic has been proven to be sensitive to changes in the isotope ratio and isotope content in the plasma. This result constitutes the first key step towards developing a CTS-based fuel ion ratio diagnostic. The next step is to routinely infer the fuel ion ratio from the obtained spectra, by fitting modeled spectra to the data taking into account all relevant parameters [22]. The comparison presented in Fig. 4 gives confidence that this goal can be achieved.

In conclusion, the results of the proof-of-principle CTSbased isotope ratio diagnostic at TEXTOR gives confidence that a novel fuel ion ratio diagnostic for ITER and future burning plasma devices can be developed. In addition, it was demonstrated that the effects on CTS spectra by IBWs and ICM for different plasma parameters and scattering geometries are consistent with our model. Simulations for ITER parameters predict that a dedicated
CTS-based fuel ion ratio diagnostic could determine the fuel ion ratio over the full plasma radius with a spatial resolution of $20 \mathrm{~cm}$ and a temporal resolution of $100 \mathrm{~ms}$ with an uncertainty of $\sigma_{R_{T}} \approx 0.15$ [23].

As a final note, one may remark that the information content in the obtained ICS in CTS spectra is high. The primary target of the present investigations was to derive information on the fuel ion ratio. However, one may expect that by refinement of the experimental setup and future development of the analysis one should be able to determine other plasma parameters such as the ion temperature $T_{i}$ with a higher accuracy than in present CTS-based methods (see, e.g., Ref. [24]).

This work has been supported by the European Communities under the contract of Association between EURATOM and Risø DTU. It was carried out within the framework of the European Fusion Development Agreement under EFDA Contract WP08-09-DIA-01-05 (VI-1) Fuel ion ratio.

*sbko@risoe.dtu.dk URL: http://cts.risoe.dk

[1] A. J. H. Donné et al., Nucl. Fusion 47, S337 (2007).

[2] I. B. Bernstein, Phys. Rev. 109, 10 (1958).

[3] M. Ono, Phys. Fluids B 5, 241 (1993).

[4] M. Stejner et al., Plasma Phys. Controlled Fusion (to be published).

[5] S. Alba et al., Plasma Phys. Controlled Fusion 35, 263 (1993).

[6] J. T. E. Grønvoll et al., Plasma Phys. Controlled Fusion 37, 1263 (1995).

[7] P. Lee et al., Phys. Rev. Lett. 49, 205 (1982).

[8] H. Park et al., Phys. Rev. Lett. 52, 1609 (1984).

[9] G. A. Wurden, M. Ono, and K. L. Wong, Phys. Rev. A 26, 2297 (1982).

[10] Y. Takase et al., Phys. Rev. Lett. 59, 1201 (1987).

[11] J. S. Machuzak et al., Rev. Sci. Instrum. 59, 1562 (1988).

[12] R. K. Richards et al., Rev. Sci. Instrum. 68, 683 (1997).

[13] E. Salpeter, Phys. Rev. 120, 1528 (1960).

[14] H. Bindslev et al., Phys. Rev. Lett. 97, 205005 (2006).

[15] S. K. Nielsen et al., Phys. Rev. E 77, 016407 (2008).

[16] S. K. Nielsen et al., Plasma Phys. Controlled Fusion 52, 092001 (2010).

[17] H. Bindslev, J. Atmos. Terr. Phys. 58, 983 (1996).

[18] H. Bindslev, Plasma Phys. Controlled Fusion 35, 1615 (1993).

[19] S. B. Korsholm et al., Rev. Sci. Instrum. 77, 10 E514 (2006).

[20] M. Stejner et al., Rev. Sci. Instrum. 81, $10 \mathrm{D} 515$ (2010).

[21] R. Schorn (private communication).

[22] H. Bindslev, Rev. Sci. Instrum. 70, 1093 (1999).

[23] H. Bindslev et al., Association EURATOM-Ris $\varnothing$ National Laboratory, Final Report EFDA Contract 041213, 2005.

[24] U. Tartari et al., Rev. Sci. Instrum. 78, 043506 (2007). 